\title{
Des franges du projet urbain au projet de frange urbaine
}

La lisière comme nouvelle figure paysagère?

From the Edges of the Urban Project to the Project of the Urban Fringe - The

Urban Fringe as a New Figure in the Landscape Architecture?"

\section{Sophie Bonin, Monique Toublanc, Pierre Dérioz et Philippe Béringuier}

\section{(Q) OpenEdition}

Journals

Édition électronique

URL : http://journals.openedition.org/paysage/10137

DOI : $10.4000 /$ paysage. 10137

ISSN : 1969-6124

\section{Éditeur :}

École nationale supérieure du paysage de Versailles-Marseille, Institut national des sciences appliquées Centre Val de Loire - École de la nature et du paysage, École nationale supérieure d'architecture et de paysage de Bordeaux, École nationale supérieure d'architecture et de paysage de Lille, Agrocampus Angers

Référence électronique

Sophie Bonin, Monique Toublanc, Pierre Dérioz et Philippe Béringuier, « Des franges du projet urbain au projet de frange urbaine », Projets de paysage [En ligne], 13 | 2015, mis en ligne le 31 décembre 2015, consulté le 05 septembre 2020. URL : http://journals.openedition.org/paysage/10137 ; DOI : https://doi.org/10.4000/paysage.10137

Ce document a été généré automatiquement le 5 septembre 2020.

Projets de paysage 


\title{
Des franges du projet urbain au projet de frange urbaine
}

\author{
La lisière comme nouvelle figure paysagère? \\ From the Edges of the Urban Project to the Project of the Urban Fringe - The \\ Urban Fringe as a New Figure in the Landscape Architecture?"
}

Sophie Bonin, Monique Toublanc, Pierre Dérioz et Philippe Béringuier

1 L'attention portée au paysage des espaces périurbains apparaît en France relativement récente. Les problèmes d'ordre paysager soulevés par le contact entre les surfaces nouvellement urbanisées et les espaces ruraux, agricoles ou «naturels », qu'elles ont colonisés, n'ont suscité l'intérêt des aménageurs que récemment, en lien avec la mise en application très progressive d'injonctions réglementaires successives (Labat, Aggeri, 2013). Dans le contexte de l'étalement urbain du dernier quart $d u x^{e}$ siècle, ces contacts semblaient généralement prendre le caractère mobile d'un «front » urbain qu'il s'agissait de maîtriser, dont le tracé s'avérait souvent temporaire et dont la forme, par voie de conséquence, n'avait qu'une importance secondaire. Métaphore guerrière, le terme de "front» souligne la confrontation entre la capacité de résistance des espaces ruraux externes et la pression conquérante de la ville, et il renvoie à une conception initiale assez linéaire de leur contact, regardé comme une césure franche entre des espaces radicalement distincts, sinon antagonistes.

2 L'objet de cet article est de documenter et d'analyser, à partir d'un corpus français, la manière de penser ce contact au fil du temps, notamment du point de vue des fonctions qu'il est susceptible de remplir et des physionomies paysagères qu'il revêt. Accompagnée par un glissement sémantique du terme de "front» vers ceux de «franges » et de « lisières » urbaines (Bonin, 2013a), cette évolution qui s'amorce dans la deuxième moitié des années 1990 tend à conférer une certaine épaisseur à cette ligne de contact, puis à l'identifier comme un espace spécifique de transition, où se déploient différentes formes de complémentarité entre la ville et ses périphéries. D'abord réfléchie en tant que bordure externe du projet urbain, la frange devient progressivement un enjeu en elle-même, qu'il est possible de travailler de manière 
spécifique, même si la mise en œuvre sur le terrain d'une telle approche reste aujourd'hui encore relativement exceptionnelle.

3 Nous voudrions dans ce texte explorer ce changement de posture. Notre hypothèse est qu'il existe un décalage, une tension entre les discours et les intentions projetés sur ces espaces, notamment en matière de planification (PLU, Scot...), et les projets d'aménagement, de gestion, de protection, effectivement réalisés. Plus concrètement, si le terme de lisière urbaine occupe une place de choix aujourd'hui dans les études préalables aux différents documents de planification d'urbanisme, ainsi que dans la conception des projets des paysagistes, il semble se diluer dans le passage au réglementaire (par exemple dans les PLU) et lors des réalisations. L'idée de lisière urbaine traverse aujourd'hui les débats, notamment entre acteurs publics de l'aménagement du territoire: c'est une intention vertueuse, louable, portée par des valeurs généreuses et actuelles, une sorte de doxa qu'il est difficile de ne pas partager. Qui pourrait aujourd'hui s'opposer à envisager une coopération entre ville et campagne ou à rapprocher ville et nature? Cependant, l'affaire se complique lorsqu'il s'agit d'incarner une telle rhétorique dans le territoire.

4 L'étude de cette prise de conscience graduelle s'inscrit ici dans le prolongement d'un programme de recherche intitulé « Paysages des franges périurbaines. Représentations, Indicateurs, Outils (PFP-RIO) ${ }^{1} »$ (Raymond et al., 2015). Ces travaux ont permis de repérer l'émergence, au sein de la problématique des paysages périurbains, d'un questionnement spécifiquement centré sur les franges, dont nous montrerons qu'il s'enracine tout à la fois dans les travaux précurseurs qui ont présidé à l'élaboration de grands schémas d'aménagement, notamment franciliens, dans la réflexion du législateur, et dans les approches pionnières d'un certain nombre de professionnels de l'urbanisme et du paysage. L'interfécondation entre ces trois sources, qui mettent en scène des acteurs différents - élus, aménageurs, experts, bureaux d'études... -, est à l'évidence très importante. L'examen des différents contextes territoriaux mobilisés à des fins de comparaison par le projet PFP-RIO - Île-de-France, ville moyenne (Narbonne), outre-mer (territoire de la côte ouest à la Réunion) - permettra ensuite de rendre compte de la mise en projet et de la mise en œuvre sur le terrain de ces nouvelles approches des franges urbaines, de l'échelle de l'agglomération (Scot) ou des communes (PLU), à celle du projet local (ZAC, lotissement). S'intéresser à ces trois régions permet de mettre en regard des situations géographiques très contrastées mais où s'appliquent les mêmes évolutions des règlements d'urbanisme, et où le discours sur l'aménagement de lisières urbaines est porté de façon variable (avec des prémices anciennes en région parisienne ; avec des porteurs de projets forts à partir de la fin des années 1990 à la Réunion ; avec une faible prise en compte en région narbonnaise).

\section{Émergence et formulation de la question des franges}

\section{Le rôle pionnier des schémas d'aménagement régionaux, en particulier franciliens}

5 Contenir le développement de la ville, en définir les contours ou du moins les limites, est l'un des objectifs poursuivis par la planification des extensions des métropoles ; ces documents de diagnostic et de stratégie politique, datés, constituent donc une source privilégiée pour étudier l'évolution des termes utilisés et des connotations qu'ils 
portent. Il convient cependant d'en relativiser la portée car outre que ces documents sont le fait d'auteurs - professionnels de l'aménagement, ingénieurs ou urbanistes - la vision qu'ils véhiculent n'est pas toujours suivie d'une application ou d'une traduction à d'autres échelles. L'appréciation du caractère consensuel ou original de ces discours par rapport à leur contexte professionnel ou historique est donc difficile, sinon impossible. Il convient de traiter nos résultats avec prudence en les rapportant à leur contexte de production, autrement dit à un corpus de discours urbanistiques historiquement situés.

6 Une lecture exhaustive des schémas d'aménagement régionaux sur nos trois terrains a été effectuée pour repérer les termes désignant les franges urbaines (au sens des extensions et des bordures des agglomérations), et relever en particulier les usages des termes de franges, de fronts et de lisières. De façon plus aléatoire selon leur disponibilité, certaines études préalables à ces documents ont été exploitées. Le cas de l'île-de-France, particulier en ce qu'il offre dès les années 1930 des plans urbains d'échelle régionale, les plus anciens de France, atteste d'une méthodologie d'emblée pionnière, véhiculant très tôt un discours sur les franges urbaines (années 1970). En Narbonnaise, des schémas analogues, plan directeur d'aménagement du littoral languedocien (1963), plan d'urbanisme d'intérêt régional (1964) et schéma directeur de la région languedocienne (1972), avaient pour objectif le développement touristique du littoral, et non la gestion des extensions urbaines, même s'ils prévoyaient des "coupures vertes» entre les unités touristiques. En l'absence de schéma directeur d'aménagement et d'urbanisme (SDAU), le premier document à l'échelle de l'agglomération narbonnaise est le schéma de cohérence territoriale (Scot) de 2007. Quant à la Réunion, son premier schéma d'aménagement régional (SAR) date de 1995. La chronologie de ces dispositifs de planification est finalement le reflet de dynamiques et d'enjeux d'urbanisation différents. En cela, le caractère pionnier des schémas franciliens est à relativiser.

7 En région parisienne, les termes d'abord utilisés sont : limites et périmètres. Le premier plan à réfléchir au-delà de Paris intra-muros, le plan d'urbanisme régional de 1934 (approuvé par décret-loi en 1939), définit un "périmètre d'agglomération » au-delà duquel il ne fallait pas étendre la zone urbanisée. Il exprime une volonté de stopper l'expansion forte et non contrôlée de l'habitat pavillonnaire en lotissements, qui marque l'après Première Guerre mondiale. Ces espaces ont pour les planificateurs des fonctions moins diverses et complexes qu'aujourd'hui (Toublanc, Bonin, 2012), l'interface espace bâti/espace non bâti n'a pas en soi d'existence spatiale et reste impensée jusque vers la fin des années 1960. Le terme de périmètre d'agglomération est repris dans le plan d'aménagement et d'organisation générale (PADOG) de la région parisienne approuvé par décret en 1960 : il était conçu comme une frontière, une enceinte, englobant les surfaces bâties existantes, incluant des interstices non bâtis, mais interdisant l'extension en tache d'huile. Il apparaît aussi dans le schéma directeur d'aménagement et d'urbanisme de la région de Paris (SDAURP) de 1965, qui n'utilise pas le mot de "front", mais celui de "frange de l'agglomération", associé à un paysage rural à préserver pour «éviter que la campagne perde l'aspect naturel qui fait son charme » (p. 237).

8 À partir des années 1970 et 1980, les termes de ceinture verte et surtout de front urbain prennent une place importante. L'idée de « front », dominante, correspond à la volonté de fixer les limites de la ville, et se retrouve dans la plupart des schémas directeurs des 
autres agglomérations. Dans le schéma d'aménagement et d'urbanisme de la région Îlede-France (SDAURIF, 1976), les limites sont plus épaisses et jouent un rôle d'interaction avec ce qui n'est plus la ville. La question des franges y est posée en des termes nouveaux et originaux, avec des « fronts externes » et des « glacis » à installer entre ces derniers et les «zones rurales périphériques contiguës ». On peut y voir une esquisse du concept de lisière urbaine tel qu'il est actuellement utilisé par les paysagistes, et le SDAURIF de 1976 est même très concret dans ses propositions d'aménagement. On y lit par exemple, à propos de la " lisière " avec les grands ensembles forestiers, qu' " afin de conserver une zone tampon nécessaire à la bonne gestion écologique de celles-ci [les forêts], une transition paysagère et fonctionnelle pourra être assurée au moyen de jardins, de mails, ou de parcs » (SDAURIF, 1976, p. 112). Il propose aussi avant l'heure l'idée d'une vocation multifonctionnelle de cet espace, associant gestion écologique, présence de jardins et pratiques de loisirs. Le schéma directeur régional de 1994 (SDRIF) n'apportera pas de concepts paysagers nouveaux, et affirme moins fortement cette multifonctionnalité potentielle des franges. Dans le schéma directeur régional d'île-deFrance approuvé en 2013, la formulation est comparable, mais les propositions sont moins précises qu'en 1976 : concernant les « espaces urbanisés [existants] à optimiser, [...] les limites de l'urbanisation existante doivent être aménagées afin de constituer un front cohérent, espace de transition et de valorisation réciproque entre ville et nature ». Le terme de lisière urbaine est absent des grands schémas directeurs cités mis à part l'expression lisière de bourg (SDRIF, 2013) - tout comme il l'est de la plupart des dictionnaires d'aménagement et d'urbanisme publiés à la même époque.

\section{Une évolution du contexte législatif favorable à l'émergence du thème des franges urbaines}

9 Quel que soit le terme retenu pour le caractériser - front, frange, interface, lisière... -, le thème spécifique du contact entre la zone urbanisée et les espaces non urbanisés qui l'entourent est, en tant que tel, à peu près absent du corpus juridique français - en dehors de la thématique spécifique des "entrées de ville²». De nombreux textes réglementaires, dans des champs très différents, ont certes édicté des règles qui s'appliquent tout particulièrement à ces espaces de contact et aux effets de proximité qu'ils engendrent : obligations de débroussaillement autour des maisons au voisinage d'étendues forestières ou de maquis (code forestier, art. L 134-6), réglementation controversée des épandages phytosanitaires à proximité des habitations (code rural, arrêté du 12 septembre 2006), prescriptions départementales et/ou locales concernant la plantation de haies ou l'érection de clôtures, etc. Mais si le droit s'intéresse aux " limites », et plus encore aux règles de « délimitation » des différentes zones urbaines, il n'a pas travaillé de manière explicite sur la forme que ces limites doivent prendre, même si cette question s'est peu à peu dessinée en filigrane dans les textes, à la croisée de trois types de préoccupations, urbanistiques, paysagères et environnementales.

10 Du côté du droit de l'urbanisme, l'impulsion donnée par la loi d'orientation foncière (LOF) de 1967 (Goze, 1999) a fait place sur cette question à une exigence de cohérence communale et intercommunale - et de précaution (loi SRU de 2000, cf. Goze, 2002), prolongée par un rejet de «l'étalement urbain » (loi ENE - Grenelle II - de 2010 ; cf. Denizeau, 2011 ; Renard, 2011). 
11 En inscrivant ce thème dans le code de l'urbanisme en tant que volet obligatoire des POS et des schémas directeurs (Labat et Aggeri, 2013), la loi Paysage de 1993 fournit un cadre plus ambitieux que celui des prescriptions de l'article R 111-21 du code de l'urbanisme (1976, modifié par décret en 2007) concernant «l'intégration » paysagère des constructions nouvelles (Guttinger, 2007). Si rien de spécifique ne concerne les franges urbaines, elles ne s'en trouvent pas moins englobées dans une injonction générale de prise en compte du paysage, qui s'accompagne de la réaffirmation de la «lutte contre l'étalement urbain et la consommation d'espaces naturels, agricoles et forestiers ${ }^{3} »$.

12 L'attention dont les franges urbaines sont susceptibles de faire l'objet découle enfin d'un troisième registre juridique, lié à la volonté du législateur d'agir en faveur de la préservation de la biodiversité en prêtant attention au bon état des "continuités écologiques » : cette préoccupation, inscrite dans le code de l'urbanisme dès l'adoption du Grenelle 1 (2009) et relayée par le projet d'identifier à différentes échelles une «trame verte et bleue », a été renforcée par la loi ENE (2010). À l'interface entre les espaces urbanisés et leurs périphéries agricoles ou "naturelles », les franges urbaines sont là encore directement concernées, notamment par la notion de "perméabilité " des aménagements ${ }^{4}$ et l'ambition d'une identification des "enjeux en matière d'intégration de la nature en ville».

Si favorables qu'apparaissent ces évolutions du droit français, la convergence des injonctions en matière de limitation de l'étalement urbain, de qualité paysagère et de continuités écologiques n'a néanmoins pas conduit à traiter directement dans la loi de la question des franges ou des lisières. Le flou et «l'absence de hiérarchisation entre [les] principes » (Melot 2011) de ce corpus législatif a ainsi poussé les acteurs publics communes, intercommunalités, PNR (Dérioz, 2013) ou CAUE - à l'expérimentation.

\section{Émergence et développement du concept de lisière urbaine}

14 Le discours sur la création et l'aménagement de lisières urbaines faisant transition, tampon voire mise en relation, entre deux milieux, se déploie en effet surtout dans la littérature grise produite par les services et les agences d'urbanisme des collectivités en synergie avec les réflexions menées par les agences privées ${ }^{5}$. Les trois terrains étudiés ont en outre montré qu'il était particulièrement présent dans des documents institutionnels non réglementaires, comme les chartes paysagères ou les plans de paysage car il apparaît comme le fruit d'une expertise demandée par les collectivités pour asseoir une réflexion politique où se mêlent projet de développement et maîtrise par la planification de certaines dynamiques à l'œuvre. C'est donc plutôt dans des documents intermédiaires, parfois préalables, là où le concepteur est sans doute le plus libre de faire des propositions nouvelles, que l'on rencontre le plus tôt et de la façon la plus aboutie l'idée de lisière comme principe d'aménagement. 


\section{Dès les années 1970, une vision du rapport ville/campagne contenant en germe l'idée de lisière}

15 Au début des années 1970, une stratégie nouvelle apparait dans la planification du développement urbain en Île-de-France, celle des zones naturelles d'équilibre qui repose sur des bases annonciatrices de l'idée de lisière. Outre une conception de l'agriculture "à même de remplir la fonction de gestion du paysage », est évoquée la perspective, au conditionnel, que la zone naturelle d'équilibre puisse « devenir, par le développement d'activités agricoles diversifiées qu'on encouragerait, le lieu de contacts et d'échanges. Ces échanges peuvent alors être source de renouvellement du respect mutuel entre deux façons de vivre et de voir les choses, entre un mode rural authentique mais rénové et un peuple citadin avide de nature mais rendu conscient de sa fragilité ${ }^{6} »$. La zone naturelle d'équilibre est aussi une composante de la «trame verte régionale ", concept alors présent dans le Livre vert comme dans le SDAURIF de 1976. Si le projet de structuration des liaisons « de la ville vers la campagne » est encore peu abouti, il y a celui de développer des pratiques d'accueil et de loisirs citadins permettant de rompre avec la « densité apparemment impénétrable du tissu urbain ${ }^{7}$ ».

Cette conception des relations ville-agriculture qui apparait pionnière dessine l'idée de lisière. Dominée par la recherche de proximités - émanant de la ville -, elle va progressivement se développer à travers une série de documents et de projets comme les chartes paysagères ou les plans de paysage portant sur des territoires de «frange urbaine ", et dans lesquels s'élabore l'idée de lisière. Aujourd'hui, dans la continuité de ces démarches, et s'appuyant notamment sur l'idée de créer des espaces de dialogue entre ville et agriculture (lisières agri-urbaines), entre ville et nature (trames vertes urbaines), on assiste à une "territorialisation» de cette vision, avec la création des territoires agri-urbains, en Île-de-France (association de la Plaine de Versailles, Terres et Cités sur le plateau de Saclay, le Triangle vert, l'association Seine Aval, etc.). Mais nombre d'autres grandes métropoles développent, à leur initiative ou à celle des territoires périphériques, le même type d'institutionnalisation ou de politique: en témoigne le recensement des dispositifs à maitrise d'ouvrage publique de "protection et de mise en valeur d'espaces urbains et périurbains agricoles ou naturels avec une partie agricole significative» conduit par l'association Terres en villes sur les 27 agglomérations adhérentes (Bonnefoy et Novarina, travail en cours de publication; Bonnefoy, 2011). L'idée de fabriquer la ville avec l'agriculture s'est progressivement imposée aux professionnels de la planification et fait aujourd'hui l'objet d'un consensus - tout au moins dans les discours - qui ne peut pas manquer de surprendre de la part d'experts a priori plutôt éloignés des questions agronomiques.

\section{Un succès incontestable dans les discours professionnels des concepteurs}

Actuellement, la lisière devient un concept largement utilisé par les concepteurs, comme le montrent les productions de l'Atelier international du Grand Paris qui a réuni paysagistes, urbanistes et architectes en 2008-2009. Plusieurs équipes ont intégré la lisière au centre de leurs propositions (LIN, Groupe Descartes, Jean Nouvel-Jean-Marie Duthilleul-Michel Cantal Dupart, Antoine Grumbach \& associés). "Intensifier les 
lisières » est ainsi devenu une des treize pistes pour « construire la ville sur la ville " formulées pour le Grand Paris.

La généalogie de la lisière comme concept de projet est délicate à affirmer de façon rigoureuse et absolue. Toutefois, depuis la fin des années 1990, deux agences paysagistes, en particulier, ont contribué à la construire et à la diffuser, avec des publications dans des revues spécialisées en aménagement. Ainsi, l'agence de Bertrand Folléa et Claire Gautier proposait, dès 1997, parmi les aménagements pour restructurer l'urbanisation réunionnaise de l'Ouest de l'île, la création de lisières urbaines (Folléa, 2011) : une « lisière urbaine... participe à l'organisation du territoire. Elle matérialise la limite d'urbanisation, forme un tampon, une interface ou un glacis entre un espace agricole ou naturel et un espace construit ${ }^{8}$ "; " des transitions sont à ménager, des lisières urbaines et agricoles à constituer, pour répondre à... [la] banalisation des paysages $^{9}$ ». La deuxième agence à voir dans la lisière une figure d'aménagement clé est celle de Michel Desvigne.

Encart 1. Extrait de Michel Desvigne, 2011

« Épaissir la lisière [...] établirait une porosité, et non une ceinture qui, fût-elle verte, ne correspondrait qu'à la dilatation d'un grillage. Ce milieu singulier ferait appel aux pratiques et aux techniques empruntées au monde de l'agriculture, et pallierait les déficits de la périphérie urbaine. [...] Coté ville, ce paysage marquerait une ouverture et la fin d'une frontière. Public, il permettrait de sortir de la logique de camp. [...] Les rues des lotissements actuellement en culs-de-sac mèneraient à des espaces partagés [...] Coté campagne, la même dilatation appliquée à la limite entre deux propriétés foncières créerait un réseau de chemins, de fossés [...], auxquels pourraient s'adjoindre quelques terrains rudimentaires (prés, vergers...) pour constituer peu à peu de vrais espaces communs. [...] L'interférence créée réconcilierait les deux mondes en leur permettant des formes de développements et d'échanges économiques de proximité. »

Appelant créativité et imagination, le principe de lisière «emprunte [au modèle du jardin] une vision idyllique et euphorisante du monde et tend à la transposer au monde réel » (Donadieu, 2009). Il se présente aussi comme un changement de regard et une réappropriation d'espaces qui «n'ont pas a priori de valeur pour un devenir tout tracé dans un urbanisme de profit foncier", mais "portent en eux une disponibilité " (Mahey, 1999). Depuis longtemps les paysagistes concepteurs se sont fait une spécialité de traiter les interfaces, de créer des paysages à partir d'une mise en valeur des limites, des franges, des interstices ( $c f$. le tiers paysage ou "fragment indécidé » de Gilles Clément, 2004), et plus récemment de revendiquer la multifonctionnalité des espaces ainsi projetés. Cette quête de mise en relation d'espaces de nature différente est pour ainsi dire consubstantielle au paysagisme: la conception d'espaces intermédiaires visant à instaurer un rapport harmonieux d'hybridation, entre la ville et la nature se retrouve déjà dans de nombreux travaux des paysagistes pionniers des années 1960-1970, comme Jacques Simon ou Jacques Sgard. Aujourd'hui, c'est au nom d'une certaine éthique (convivialité, mixité, lien social, «vivre ensemble »...) conjuguée aux valeurs du développement durable (Courcy, Bordreuil, 2012) qu'ils considèrent que la frontière entre espace urbanisé et espace non urbanisé "doit» être pensée, transformée, aménagée et devenir lisière. 
20 De nos jours, lorsque les concepteurs convoquent cette figure d'aménagement à l'échelle de la planification, leur objectif est de recoudre un territoire morcelé, éclaté, en transgressant les limites, voire les frontières, qui le traversent. Il n'est ainsi pas anodin que le mot « lisière " ait comme premier sens la bande constituant le bord d'un drap, c'est-à-dire un terme de couture, comme il y en a de nombreux autres dans le vocabulaire des paysagistes (ourlet, couture, tissage, trame...). Lorsqu'ils descendent à l'échelle d'un projet d'aménagement et de propositions concrètes, ils se réfèrent à une vision continue du rapport de la ville avec son environnement proche. Leur modèle est celui de la symbiose - association et porosité - avec l'idée que chaque partie trouve des avantages dans cette cohabitation spatiale. Quand l'espace qui jouxte la ville a une vocation agricole, les propositions des concepteurs défendent l'idée d'une pérennisation et même d'un développement de l'agriculture aux portes de la ville, au nom de la multiplicité des fonctions que celle-là est censée remplir : alimentaire, économique, productrice d'emplois, environnementale, pédagogique, paysagère et patrimoniale, récréative.

21 Le principe de lisière véhicule ainsi une utopie à la fois sociale, politique et environnementale. Toutefois, comme toute vision idéale, elle peine à s'inscrire dans la réalité paysagère des territoires, où l'enjeu demeure souvent la maîtrise de l'étalement urbain.

\section{Les franges urbaines en projet dans trois contextes urbains différents}

22 Si nos trois régions d'étude montrent qu'une certaine percolation s'observe dans les Scot les plus récents, trouver dans les PLU, c'est-à-dire à l'échelle communale, la formulation de recommandations pour aménager des lisières urbaines est bien plus hasardeux. Au-delà de cas particuliers, il n'est pas facile de saisir une évolution générale dans la durée qui tendrait vers une généralisation de ce type de recommandation à toutes les communes. En effet, les mises en œuvre, inégalement précoces et inventives, sont souvent en deçà de l'intention contenue dans le concept de « la lisière urbaine ».

\section{De l'intention à l'inopérabilité en Île de France-Plaine de Versailles}

Si les acteurs en charge de la planification urbaine en Île de France (Thibault, 2010) s'approprient largement le discours et la rhétorique paysagistes, en particulier dans la conception des grands schémas, on peut s'interroger sur leur traduction dans les documents d'urbanisme à une échelle communale ou intercommunale et dans des aménagements portés par la puissance publique et/ou des aménageurs privés (promoteurs immobiliers...) à l'échelle de l'îlot ou du quartier. Du fait notamment de l'ancien statut de zone naturelle d'équilibre de la Plaine de Versailles dans les années 1970, l'hypothèse d'une certaine percolation contemporaine de l'idée de création de lisières agri-urbaines y a été tout particulièrement examinée. Ce territoire à une vingtaine de kilomètres à l'ouest de Paris est en effet soumis à une forte pression d'urbanisation, mais dispose aussi de protections fortes de son espace agricole : un site inscrit de 2000 ha de surface agricole, au titre notamment du prolongement de la perspective du château de Versailles, et une gouvernance originale du territoire plus 
large, de 24 communes, où se retrouvent élus, agriculteurs et particuliers dans une association patrimoniale (Bonin, 2013b). La proximité physique avec l'École nationale $\mathrm{du}$ paysage de Versailles, formant des paysagistes concepteurs, est un facteur supplémentaire. Il n'est donc pas surprenant de retrouver le terme et l'idée de lisière mettant en relation ville et agriculture à travers l'aménagement des franges urbaines, exprimé à l'échelle de ce territoire. Cela se traduit dans une charte paysagère conduite par l'association patrimoniale, datant de 2013, annexée au Scot qui l'a suivie de près (2014), dont un enjeu important est de «cultiver un dialogue paysager entre espaces agraires et espaces bâtis ». Assez concrètement, le plan d'aménagement et de développement durable (PADD) du Scot Gally-Mauldre (2014, p. 17) - qui n'inclut que onze des vingt-quatre communes signataires de la charte paysagère - annonce des «règles de gestion des lisières urbaines afin d'organiser des transitions paysagères entre les espaces urbains et les espaces agricoles ou les espaces naturels ». Pourtant, si les orientations d'aménagement de certains PLU de la Plaine de Versailles mentionnent bien la constitution d'une «lisière plantée » ou d'une "trame [verte]» en limite des zones d'extension urbaine prévues, ce n'est pas le cas de tous les PLU, même récents, et le principe tel que nous l'avons évoqué précédemment est très amoindri dans ses ambitions de mise en dialogue. On peut même faire des constats, dans la mise en pratique à l'échelle du quartier cette fois, d'une inefficience de ce principe. C'est le cas pour des nouveaux quartiers en frange, qui devraient au contraire devenir le lieu par excellence de concrétisation de ces idées. Sur deux projets qui sont entrés en chantier en 2014-2015, la ZAC Charles-Renard à Saint-Cyr-l'École (25 ha), et le quartier des Hauts du Moulin à Villepreux (13,5 ha), les promoteurs et les aménageurs ne se saisissent pas de cette idée de lisière. La maîtrise d'œuvre est passée à des opérateurs (AFTRP pour la ZAC Charles-Renard) et à des promoteurs (Bouygues Immobilier dans le premier cas, Kaufman et Broad dans le second) dont la communication n'envisage pas l'aménagement de transition ou l'instauration d'un dialogue avec en particulier le monde agricole. 
Figure 1. Limite entre l'espace agricole et le nouveau quartier des Hauts du Moulin à Villepreux

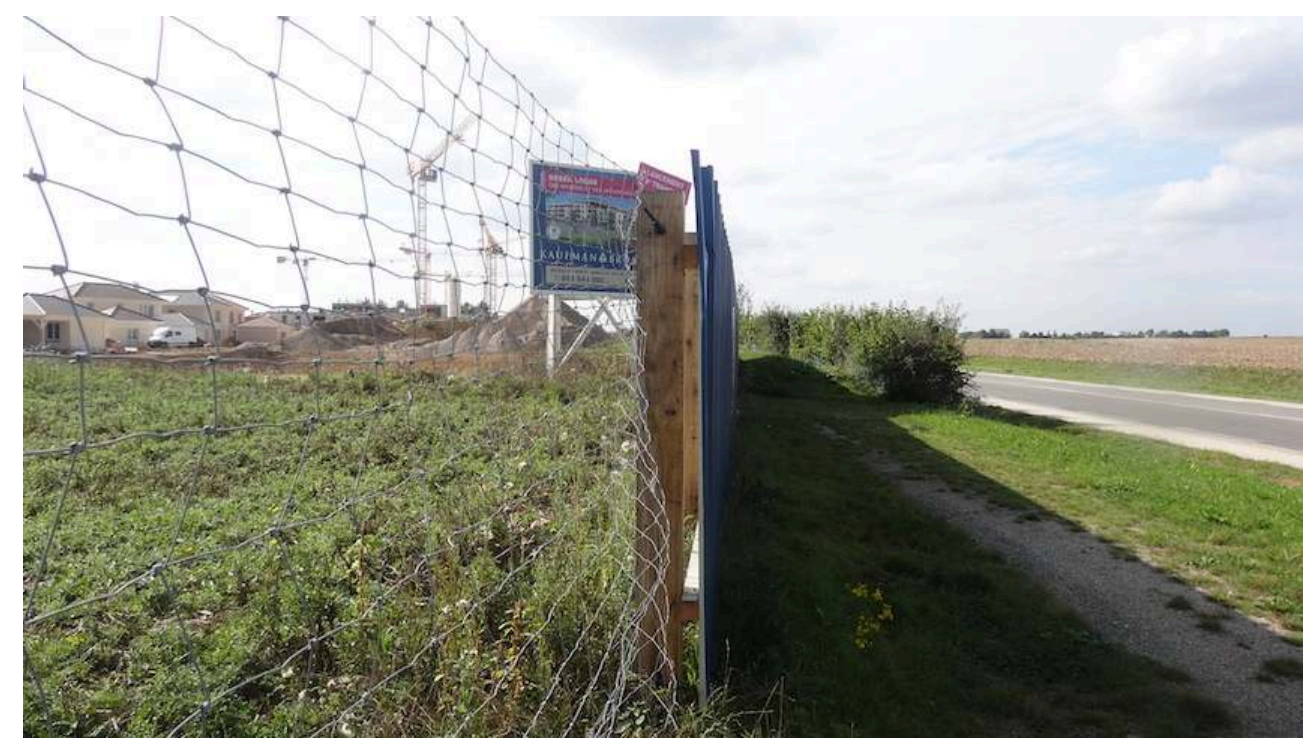

Cette extension urbaine était dans les orientations du SDRIF : la communication met en avant l'espace boisé situé à proximité, mais ne dit rien de la plaine agricole qui est pourtant à cet endroit un site classé. L'enjeu paysager principal dans la communication faite par la mairie porte sur une

"intégration réfléchie pour ne pas nuire aux zones déjà bâties », qui a eu comme exigence que les logements les plus hauts et denses soient en limite, et les logements pavillonnaires à l'intérieur du quartier (http://www.villepreux.fr). Pour Kaufman et Broad, les « belles vues » sont sur les jardins intérieurs et les allées arborées. Enfin, le PLU évoquait une "frange paysagère à créer » : il en résulte une haie en bord de route, qui « sécurise » une piste cyclable, par rapport à une route à circulation rapide de toute façon infranchissable à pied.

Source : Sophie Bonin, octobre 2014.

Beaucoup des PLU des communes concernées par la charte paysagère ou par le Scot sont antérieurs à 2011, ce qui ne permet pas d'argumenter sur la pression réglementaire. Mais la charte paysagère aurait pu être prise en compte par la maitrise d'ouvrage, voire par les aménageurs eux-mêmes, qui pourraient avoir un intérêt à valoriser ce paysage agricole, d'autant que cette plus-value possible n'a pas forcément un coût élevé. Il pourrait simplement être question d'ouvertures visuelles et d'accessibilité. L'hypothèse envisagée pour comprendre cette absence de mise en œuvre, mais que l'on n'a pas pu tester auprès des aménageurs, est que la reconnaissance paysagère de l'espace agricole n'est pas partagée, peut-être pas encore, par le public des futurs propriétaires ou locataires des logements: c'est une reconnaissance qui serait confidentielle, et qui ne convainc pas, pour l'instant du moins, le dernier maillon des constructeurs de la ville dans ces milieux périurbains.

\section{Itérations entre échelles, entre conceptualisation et planification des projets à la Réunion}

Un terrain bien différent nous a intéressés, le territoire de la côte ouest (TCO) de lîle de La Réunion, parce que, dans cette communauté d'agglomération, la lisière urbaine comme figure de projet et d'aménagement a trouvé un terreau favorable à son énonciation dans la planification urbaine.

Dominé par de la savane et entaillé par de grandes ravines, ce territoire très attractif, proche de la capitale régionale Saint-Denis, est marqué par une croissance 
démographique et urbaine forte, amplifiée par la mise en service de la route des Tamarins en 2009 et le projet ILO (Irrigation du littoral ouest, 1998) de basculement des eaux d'est en ouest. Outre le développement de la surface en canne à sucre en lieu et place de la savane, ILO augmente aussi les capacités du territoire à s'urbaniser. À la Réunion, et en particulier sur cette partie de l'île, l'urbanisation récente et massive est à l'origine de transformations paysagères sans précédent. L'autre particularité de ce territoire est la nature de l'espace non bâti : ravine ou savane. Milieu spécifique de parcours pastoral, la savane a amorcé un recul significatif du fait de l'urbanisation et de la progression de la canne à sucre, et devient aujourd'hui le cœur d'une controverse opposant défenseurs de l'environnement et usagers (éleveurs, promeneurs...), et tenants du développement urbain. Pour tenter de maîtriser ces différentes dynamiques aux répercussions territoriales et paysagères jugées problématiques par un certain nombre d'acteurs (associations, parc national, communauté d'agglomération...), les élus et les services de l'État ont engagé sur le TCO depuis environ deux décennies une réflexion approfondie sur la planification, l'aménagement et la gestion des territoires et des paysages avec pour particularité de mobiliser l'idée de lisière urbaine comme concept de projet. En 2009, un schéma intercommunal d'aménagement des lisières urbaines, premier du genre en France, précède le Scot de 2011. Les idées du premier vont féconder le second, considérant les lisières urbaines, au même titre que les ravines, comme des espaces naturels aptes à offrir « des aménités de la vie » pour les populations, donc à valoriser (encart 2). Outre des fonctions sociales, paysagères et urbanistiques, elles sont aussi présentées comme pouvant jouer un rôle écologique, et contribuer par conséquent à la trame verte et bleue (encart 3).

Encart 2. Extrait du rapport de présentation, Scot du TCO, 2011, p. 79

« Les lisières urbaines à identifier et à aménager peuvent assurer trois fonctions, plus une, qui vont contribuer à améliorer l'agrément de vie des résidents et visiteurs de l'Ouest de la Réunion :

- Faciliter la politique de protection des espaces agricoles et naturels. En effet, sur les immenses pentes du grand planèze on peut presque dire que tout établissement humain (habitat, équipement, zone d'activités, réseaux) peut prendre place en tout lieu. Seul l'impératif contractuel et réglementaire fort que constitue le programme de l'irrigation du littoral ouest (Pilo) a permis, il est vrai, d'enrayer quelque peu la tendance à l'ubiquité sans frein de l'urbanisation. Mais au-delà de la contrainte à l'urbanisation que peut, à juste titre, représenter le Pilo, n'est-il pas venu le temps de " positiver » et de produire une meilleure relation entre l'homme, la nature et la ville ? Dans ce contexte, la lisière urbaine matérialise une limite plantée et permet d'identifier visuellement respectivement l'espace urbain et l'espace agricole.

- Offrir des espaces publics de proximité. Avec le développement de l'urbanisation sous des formes déjà resserrées et qui le seront de plus en plus, le besoin d'espaces publics naturels de proximité va croitre. Les lisières urbaines sont une des réponses en accueillant des lieux et des petits équipements pour la promenade et le sport-loisir. 
- Permettre l'implantation de jardins familiaux créoles. La civilisation créole à la Réunion comme aux Antilles est, même urbaine, une civilisation végétale. Au regard de l'intensification de l'urbanisation, le jardin créole dans les tissus urbains va devenir plus difficile soit à préserver, soit à programmer. Dès lors, les lisières urbaines pourraient être une bonne alternative avec des jardins créoles à la disposition des habitants du voisinage. Au-delà de ces fonctions relevant proprement des aménités de la vie, les lisières urbaines peuvent également avoir une fonction écologique.

- Contribuer à préserver les milieux naturels marins, notamment le lagon et les récifs de l'Ouest localisés au droit de la planèze de l'Ouest.

Encart 3. Extrait du rapport de présentation, Scot de TCO, 2011, p. 88

Aux ravines s'ajoutent les lisières urbaines qui, au-delà de leur contribution aux aménités paysagères, peuvent/doivent jouer un rôle écologique de protection des cours d'eau, des bassins-versants et du lagon en minimisant les phénomènes de ruissellement et d'érosion des sols.

Au regard des courbes de niveau, les lisières urbaines globalement localisées en parallèle et les ravines évidemment localisées en perpendiculaire peuvent constituer une véritable trame assurant des continuités et proximités en faveur de la circulation des espèces et en évitant la fragmentation des milieux naturels.

La loi SRU (2010) imposant les Scot aux PLU, l'idée de lisière urbaine, énoncée de façon explicite dès 2009 à l'échelle intercommunale, irrigue-t-elle les projets locaux ? L'analyse a porté sur la principale commune du TCO, Saint-Paul, son PLU (2012) et son projet d'extension urbaine, la ZAC Renaissance III, dans le quartier de plateau Caillou ${ }^{10}$. Trois documents constitutifs du PLU, quoique de statuts différents, ont été examinés : le Rapport de présentation, le PADD qui définit des orientations générales et les Orientations d'aménagement et de programmation (OAP) qui intègrent des dispositions plus précises.

En l'occurrence, le Rapport de présentation utilise la terminologie "lisières urbaines » en se référant à la charte paysagère élaborée à l'échelle du TCO (2007) et insiste sur leur richesse potentielle, support de multifonctionnalité. Si le PADD du PLU ne reprend pas le vocable et passe même sous silence la question des relations entre espaces bâtis et espaces non bâtis, l'idée revient dans le document OAP infuser le projet d'extension urbaine ZAC Renaissance III. Cependant, tandis que le plan-guide de la charte paysagère préconisait d'accompagner ce projet d'urbanisation par l'aménagement de lisières prenant la forme de «bandes plantées " afin de composer « un front urbain en dialogue avec la savane", préconisations qui seront reprises en 2009 dans le schéma intercommunal des lisières, la version approuvée du projet de la ZAC telle qu'elle apparait dans le document OAP (2012) propose un dispositif appauvri, bien en deçà des objectifs de départ. Il entérine certes une transition verte, mais discontinue, à vocation uniquement écologique, et nommée trame verte. Le principe de multifonctionnalité semble s'être dissous: les fonctions sociales (mise en relation de deux mondes, production d'aménités pour les riverains), esthétiques et spatiales (organisation du territoire...) ont pour ainsi dire disparu, laissant place au seul discours écologique, conformément à la prégnance des injonctions environnementales. Le contenu de la ZAC 
Renaissance III montre clairement l'ampleur de la déperdition de l'idée de lisière lors du passage de la planification intercommunale à la conception d'un nouveau quartier. Après avoir examiné de près l'ensemble du PLU de Saint-Paul et les autres documents de planification produits sur le TCO, il s'avère qu'il ne s'agit pas là d'un cas isolé. Si l'idée pénètre les différentes échelles, c'est au prix d'une dilution et d'une perte de contenu.

Figure 2. Des principes du schéma intercommunal des lisières aux OAP du PLU de Saint-Paul, des ambitions en recul pour les lisières urbaines de la ZAC Renaissance III

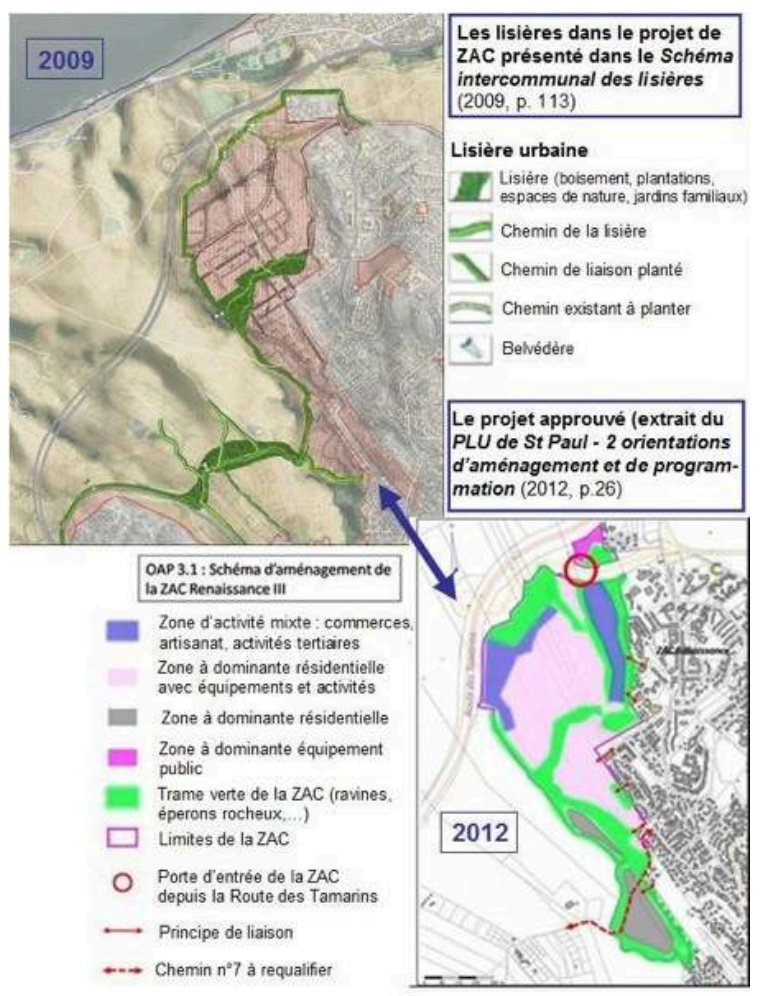

Jusqu'alors, le principe d'aménager des lisières n'a donné lieu qu'à de très rares réalisations concrètes et seulement dans des conditions particulières. Les seuls aménagements repérés in situ ont plutôt été réalisés sur les interfaces espaces bâtis/ espaces « naturels » (encarts 4 et 5 ) et non entre espaces construits et espaces agricoles (canne à sucre, vergers, horticulture...). 
Encart 4. Un travail sur les lisières réservé aux interfaces espaces bâtis/espaces naturels : exemple 1 , la ZAC de la Cocoteraie

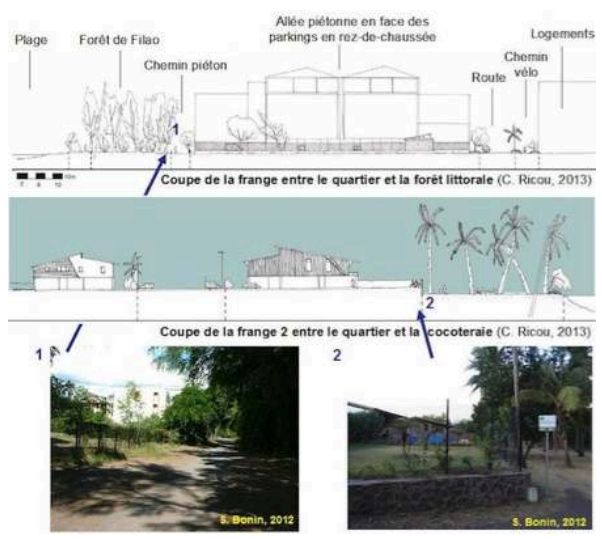

Cette extension urbaine, livrée en 2010, est située dans le quartier de la Poudrière à Saint-Paul, au bord de la mer et en limite de l'étang de Saint-Paul classé en réserve naturelle. Elle a été implantée sur un ancien terrain vague bordé par une cocoteraie et une forết domaniale de filaos qui ont éte l'une et T'autre préservées en aménagées pour le loisir et la détente (balade, jogging...). Ouvertes au public, notamment à travers le sentier du littoral, elles constituent un espace tampon avec la mer a Touest et retang au sud. La cocoteraie, espace «naturel $n$ de production arboree a la rentabilite hypothetique, et la foret de fliaos ne sont pas incluses dans

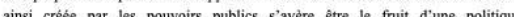
volontriste visant à aménager les bords de mer à des fins de loisirs, et pour we population urbaine grandissante, C'est aussi une politique originale qui contribere à un changement culturel puisque, traditionnellement, le rivage n'est pas fréquenté par la société réunionnaise. Longtemps, celle-cí a été (êt est toujours attirée vers les Hauts, perçus comme le territoire par excellence du ressourcement. La lisière aménagée de la ZAC de la Cocoteraie est devenue un espace public qui

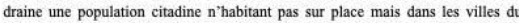
littoral. L'appropriation par les riverains en est ténue, ils ne la fréquentent pas au quotidien, et la vivent davantage comme un écrin de leur lieu de vie. En cela, la lisière ne remplit pas sa promesse de mise en relation des riverains avec leur milieu.

\section{Encart 5. L'idée de la lisière-décor : exemple 2, Piton Saint-Leu}

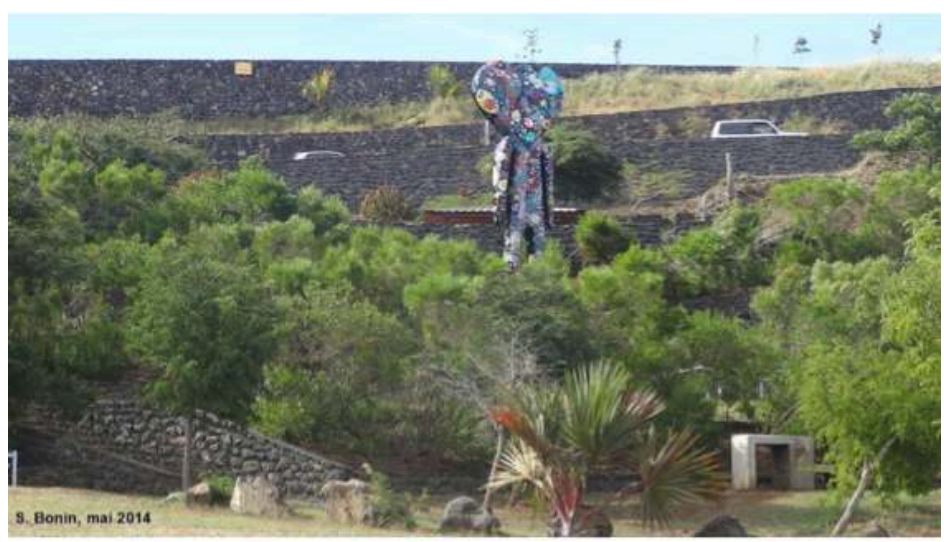

Un second cas a été repéré sur la commune de Piton Saint-Leu. Mis en cuvre lors de la construction de la route des Tamarins dans le cadre du $1 \%$ paysage lié aux infrastructures routières, l'aménagement se déploie, là encore, sur du foncier public, conquis sur un territoire de savane aux usages aujourd'hui diversifiés : randonnées pédestres et équestres, cueillette, parcours pour le bétail (éleveurs « informels » de bovins et caprins), restauration naturaliste. La savane, territoire ouvert et accessible, n'étant pas reconnue comme un espace ayant une vocation agricole, la lisière aménagée par les pouvoirs publics n'instaure pas de fait un dialogue entre ces deux univers, peut-être même crée-t-elle une distance entre eux deux, dans la mesure où elle favorise des usages urbains de ce milieu -

contemplatifs et récréatifs -, érige celui-ci en un décor naturel paré de qualités écologiques et paysagères et, ce faisant, au mieux ignore, au pire s'oppose aux pratiques pastorales résiduelles.

30 Sur le TCO, finalement, la lisière urbaine demeure avant tout une figure discursive. Mise à l'épreuve des réalités du terrain, elle se réduit à quelques cas particuliers qui 
participent peu à rapprocher les univers agricole/naturel et urbain. Une telle volonté n'est d'ailleurs peut-être pas adaptée, tant la population réunionnaise a conservé un mode d'habiter rural où cueillette, petit élevage, jardinage, plantations vivrières, médicinales, horticoles trouvent leur place d'abord dans l'espace privé, y compris dans les nouvelles extensions urbaines tout comme dans l'espace public ou collectif interstitiel, tombé en déshérence ou en attente d'affectation. Bien qu'à La Réunion, la mise en œuvre des extensions urbaines reste marquée par la création de coupures franches avec les espaces « ouverts » environnants, il n'est pas rare que les riverains s'approprient de façon spontanée et provisoire les franges pour avoir qui un potager, qui un petit élevage, qui une volière... brodant ainsi des lisières à leur image.

Figure 3. L'espace privatif d'un logement locatif de la ZAC Renaissance III transformé en « Kour » (ou la prégnance du modèle du jardin créole)

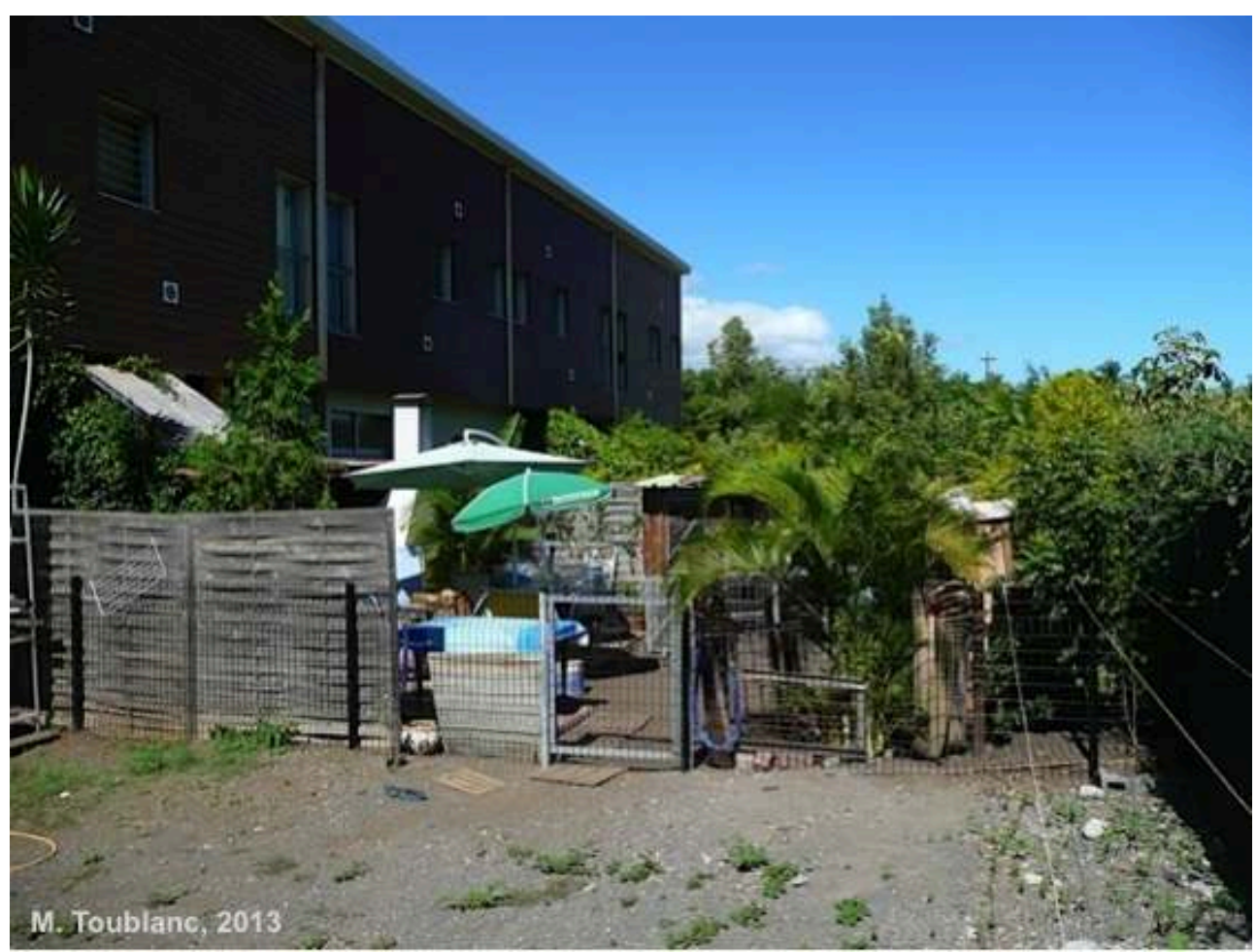


Figure 4. Vue de l'intérieur du quartier vers la savane, par un accès latéral

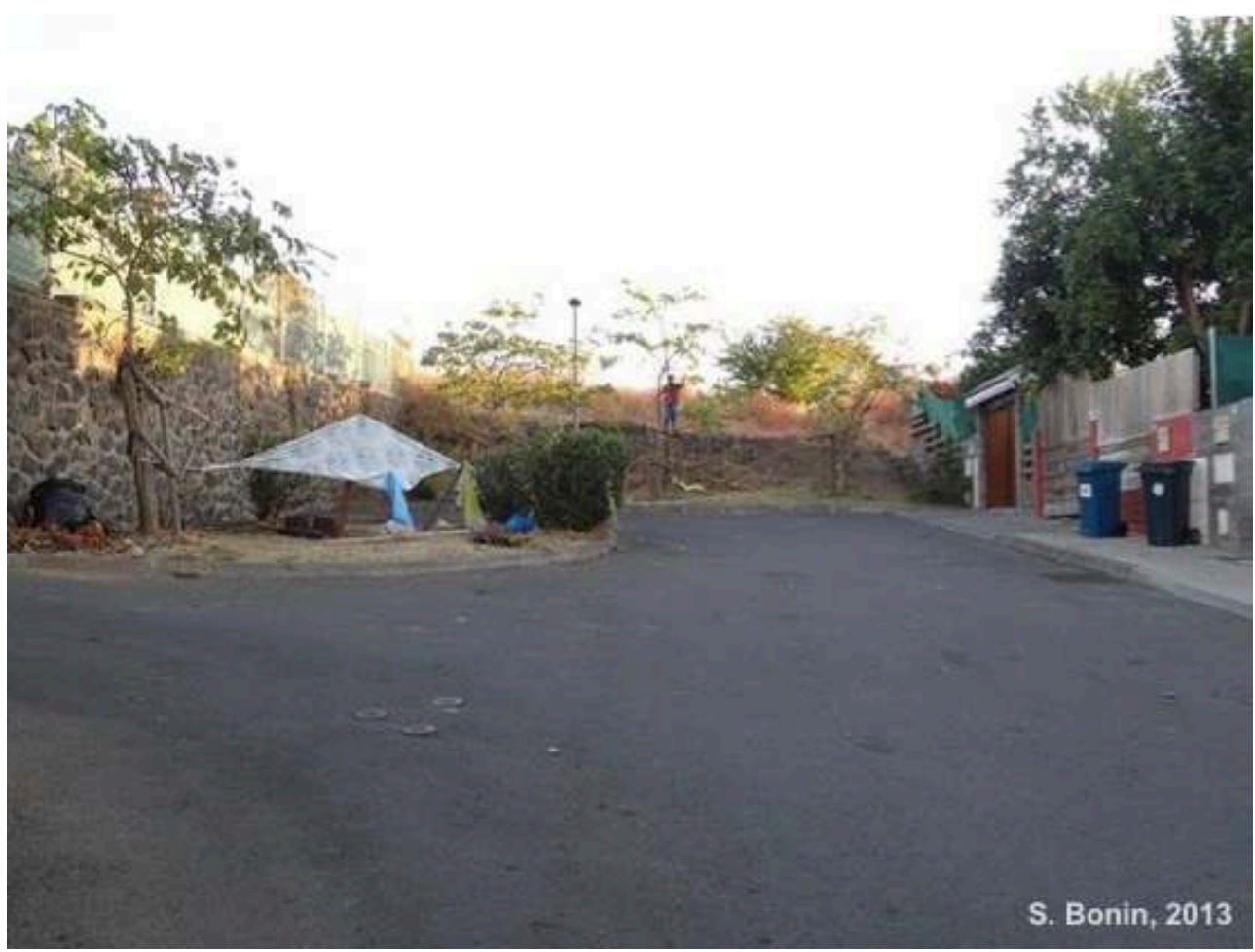

Figure 5. Les installations précaires d'une lisière habitante entre espace bâti et savane ; à droite, un poulailler

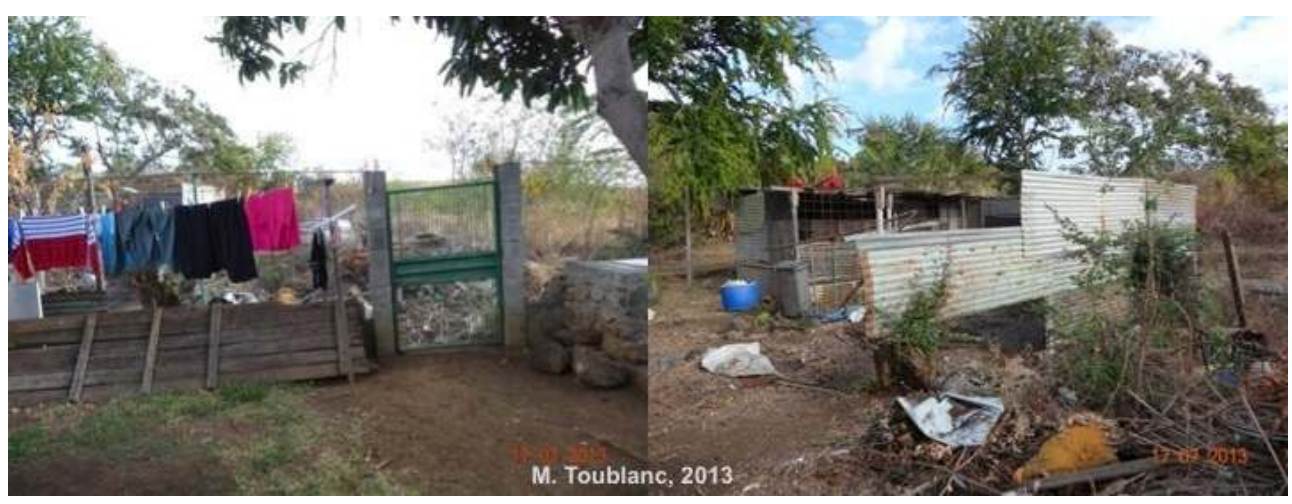

\section{Du « laisser-bâtir » à la découverte des franges en Narbonnaise : une prise de conscience tardive enclenchée par les documents d'échelle intercommunale}

31 Caractéristique du phénomène de métropolisation qui caractérise les plaines $d u$ Languedoc depuis plus de trente ans, la spectaculaire croissance démographique qui a affecté le Grand Narbonne ${ }^{11}$ s'est majoritairement traduite par le développement d'extensions résidentielles plutôt compactes en périphérie du bâti ancien des villages satellites de Narbonne, selon un modèle « archipélagique » qui a démultiplié le linéaire de franges urbaines (en tout, $450 \mathrm{~km}$ cumulés en 2008). La production en série de lotissements pavillonnaires accolés, fermés sur eux-mêmes, et qui dans la plupart des cas mettent en contact l'espace agricole ou l'espace «naturel » périphérique avec des 
quartiers pavillonnaires, découle de trois ordres de facteurs: de fortes contraintes spatiales (zones inondables et espaces protégés étendus), le bas niveau de revenu moyen des populations audoises, qui a très souvent conduit les ménages candidats à la construction d'une maison individuelle à opter pour les solutions les plus économiques, et des stratégies municipales favorables à l'ouverture de droits à construire, dans un contexte de crise et de recul de la viticulture qui favorise la libération de foncier.

Figure 6. Des franges contraintes, des fronts d'urbanisation limités : exemple de Vinassan

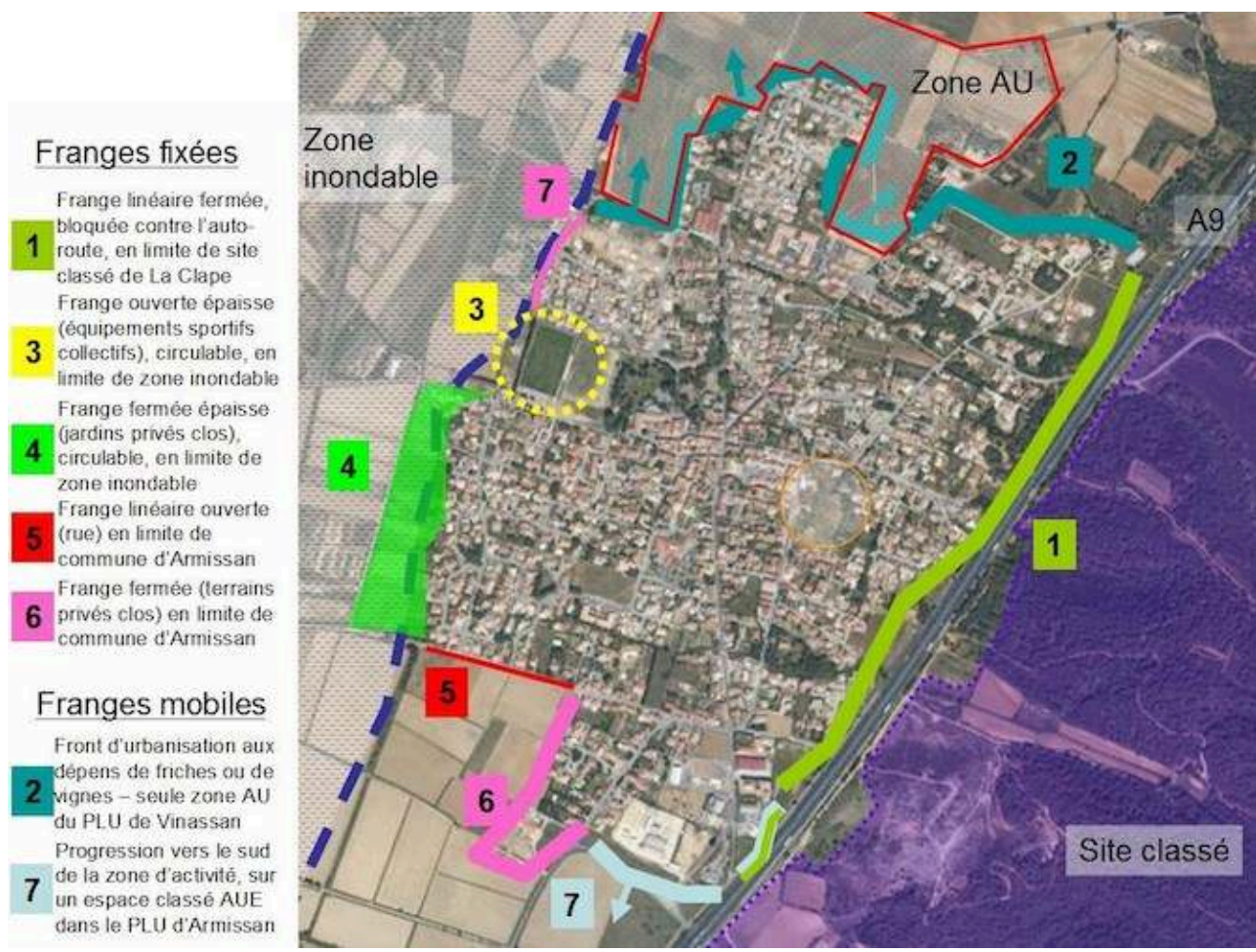

La tentative de typologie des franges urbaines qui a été réalisée sur les trois terrains du programme PFP-RIO (Raymond et al., 2015) a souligné en Narbonnaise l'importance prise par les franges " fixées », comme ici à Vinassan, au nord-est de Narbonne, où la fixation des franges découle aussi bien de contraintes physiques (autoroute) que de contraintes de protection (site classé) ou de risque (inondation). Franges durablement bloquées (Coursan) et franges mobiles (Portel-des-Corbières), pourtant, ne présentent pas nécessairement des physionomies très dissemblables, à la rencontre entre aménagements publics et microaménagements privés. 
Figure 7. Coursan, 2014

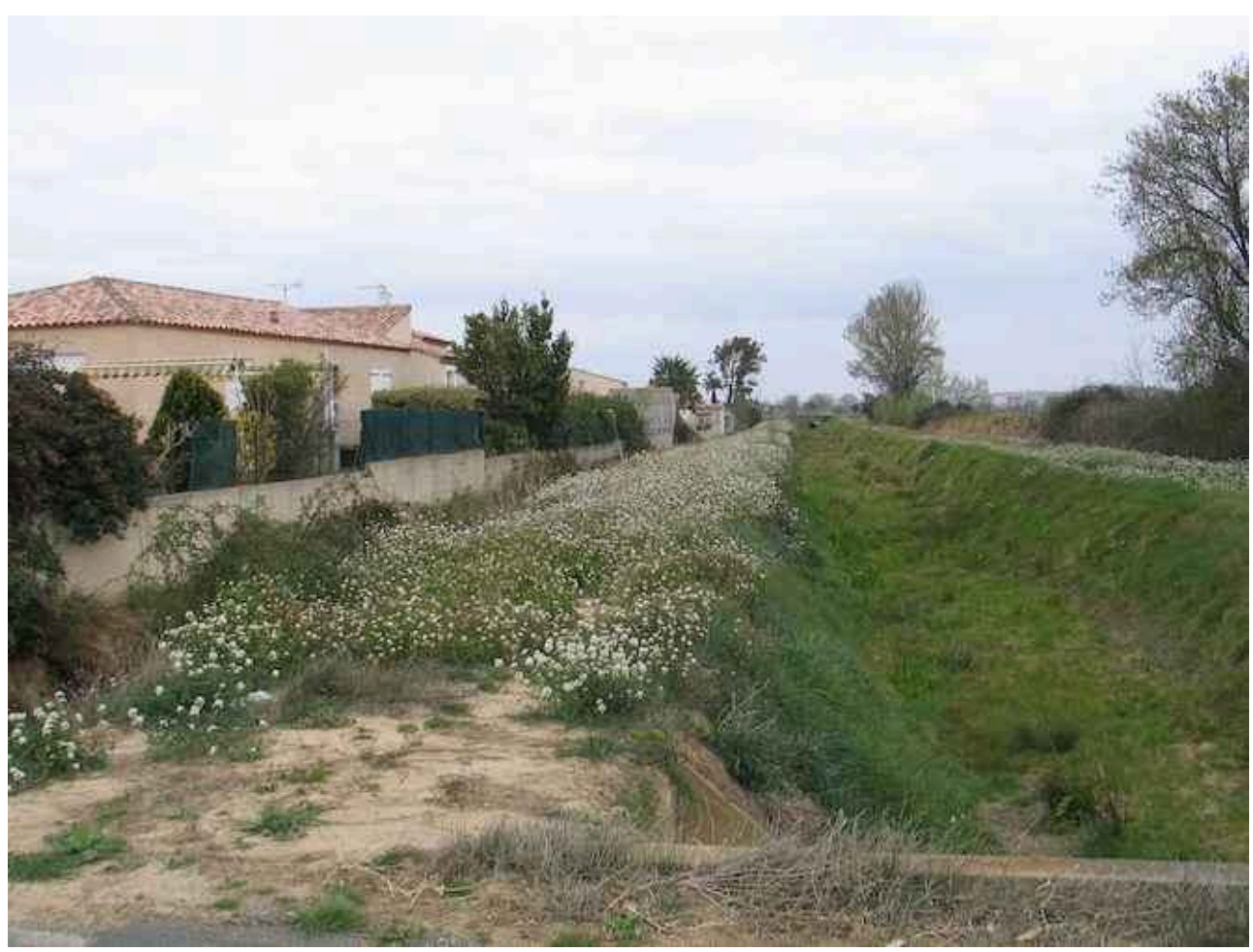

Déjà en zone potentiellement inondable, les maisons individuelles alignent les murs d'enceinte et/ou leurs haies végétalisées le long du large fossé de protection qui limite le village du côté est.

Figure 8. Portel-des-Corbières, 2013

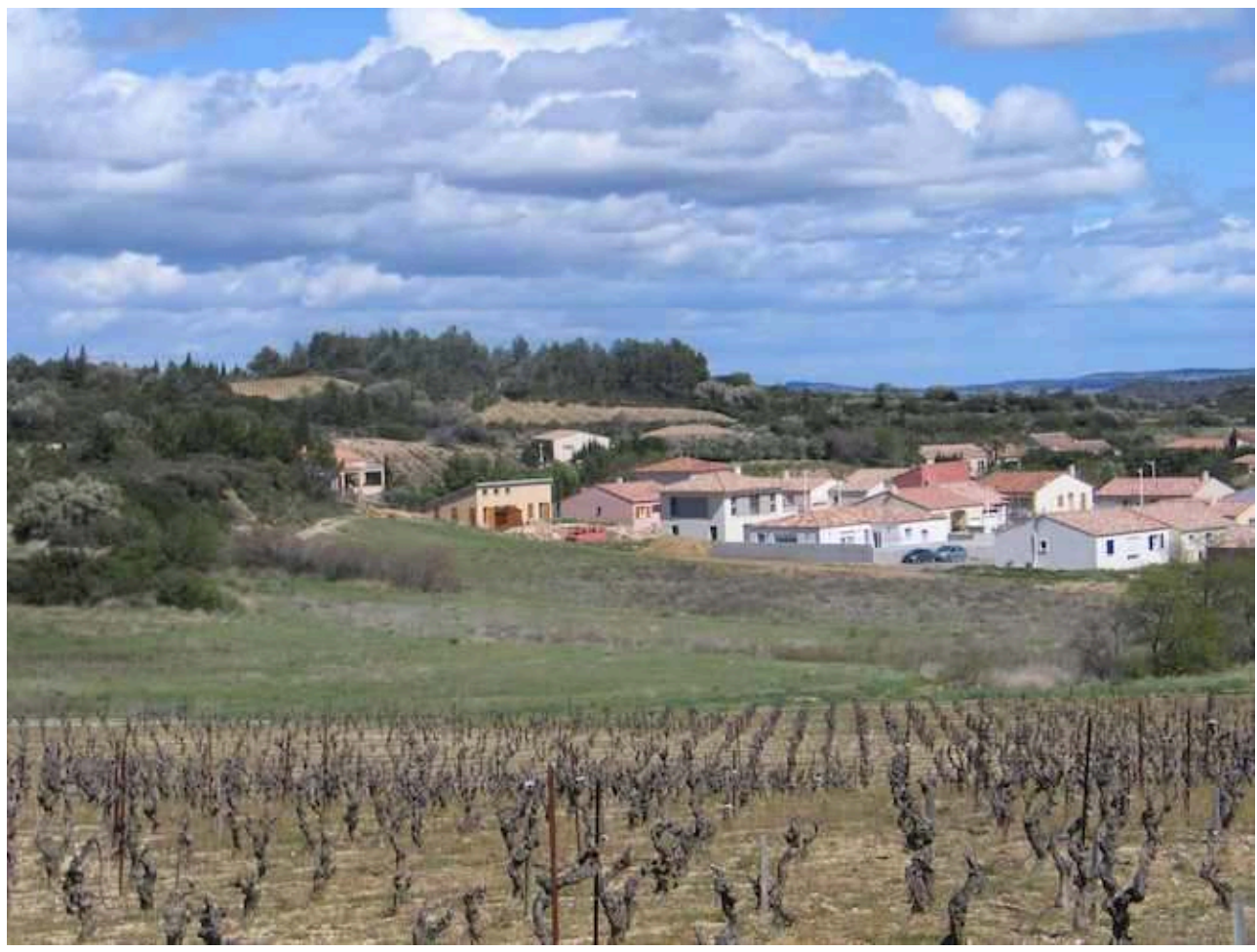

Les murs de parpaings bruts des nouveaux pavillons sont longés par la voie (encore un simple chemin) qui desservira les maisons à venir sur la friche constructible (ancienne vigne) qui s'inscrit dans leur prolongement. 

gestionnaires, les aménageurs et les politiques en terme de dynamique spatiale, tant du point de vue de la "création" de foncier urbanisable que de celui du constat du « repli » viticole. L'introduction d'une approche paysagère est très récente, une dizaine d'années à peine, et le fait de s'intéresser à la limite elle-même en tant que lieu et paysage singuliers l'est plus encore. L'examen du contenu des premiers POS montre que les préoccupations paysagères s'en trouvent largement absentes, du moins au-delà du rappel dans les règlements des prescriptions concernant «l'intégration" des constructions nouvelles. Il faut le choc des inondations de novembre 1999 pour remettre en cause ce positionnement et faire apparaître la nécessité d'une réflexion d'ensemble, au moment où la Loi SRU (2000) enclenche le processus de conversion des POS en PLU. L'élaboration des PADD, où les enjeux paysagers apparaissent comme un passage obligé, implique alors le recours à des bureaux d'études dont certains seront réellement force de propositions. Cette entrée du paysage dans les documents d'urbanisme communaux est en lien avec l'approbation des premiers documents-cadres à l'échelle intercommunale: la charte du PNR de la Narbonnaise en Méditerranée (2002), qui entend accompagner les communes pour "prendre en compte le paysage dans les zones d'extension urbaine » (Dérioz, 2013), et sert en bonne partie de référence au premier Scot (2006) sur les thèmes environnementaux et paysagers.

Certes, le recours à des termes spécifiques (franges, lisières) pour désigner l'enjeu des limites, attesté dans ces documents, ne signifie pas qu'il existe à son endroit un consensus étendu : après avoir suivi la réalisation de plusieurs PLU, le PNR tend à se désengager de cette thématique, comme il recule en 2013 sur son projet de charte paysagère pour le Piémont des Corbières maritimes (où les transformations sont les plus actives, au sud de Narbonne), dont l'un des enjeux touchait à «la gestion des interfaces entre franges agricoles et urbaines [qui] doit permettre de limiter les confrontations brutales en incitant aux transitions et à la découverte, d'offrir un espace de respiration, un premier plan aux silhouettes villageoises ${ }^{12} »$.

Pourtant, sans que la tendance soit générale, le thème des franges urbaines (ou lisières, bordures, interfaces...) semble avoir percolé, en une petite dizaine d'années, au sein du débat public des projets urbains d'échelle communale. La nécessité d'impliquer les citoyens dans les démarches a conduit dans le même temps à s'intéresser à leurs pratiques spatiales, dans des territoires communaux dont le rythme de transformation tend à se ralentir, et où de nombreuses franges urbaines semblent plus ou moins définitivement fixées, et pour certaines appropriées ou utilisées. De plus, l'intégration progressive des préoccupations liées à « la préservation et la remise en bon état des continuités écologiques» (art. L 121-1 du code de l'urbanisme, Grenelle de l'environnement) a également contribué à focaliser l'attention sur les modalités de contacts entre milieux de différente nature, donc, d'une certaine manière, sur les franges. Là où elle existe (Montredon-des-Corbières, Narbonne, Coursan, Sigean...), cette réflexion sur les franges s'est construite entre élus locaux, bureaux d'études et (souvent) techniciens du Grand Narbonne ; elle tente ainsi d'articuler entrée écologique (trames vertes), entrée fonctionnelle (gestion des risques, modalités de circulation, pratiques de loisirs) et entrée paysagère. Relativement précises et localisées, les déclarations d'intention des documents d'urbanisme, à la différence des injonctions générales des documents-cadres de type Scot, devraient peu à peu trouver une traduction concrète sur le terrain.

Projets de paysage, 13 | 2015 


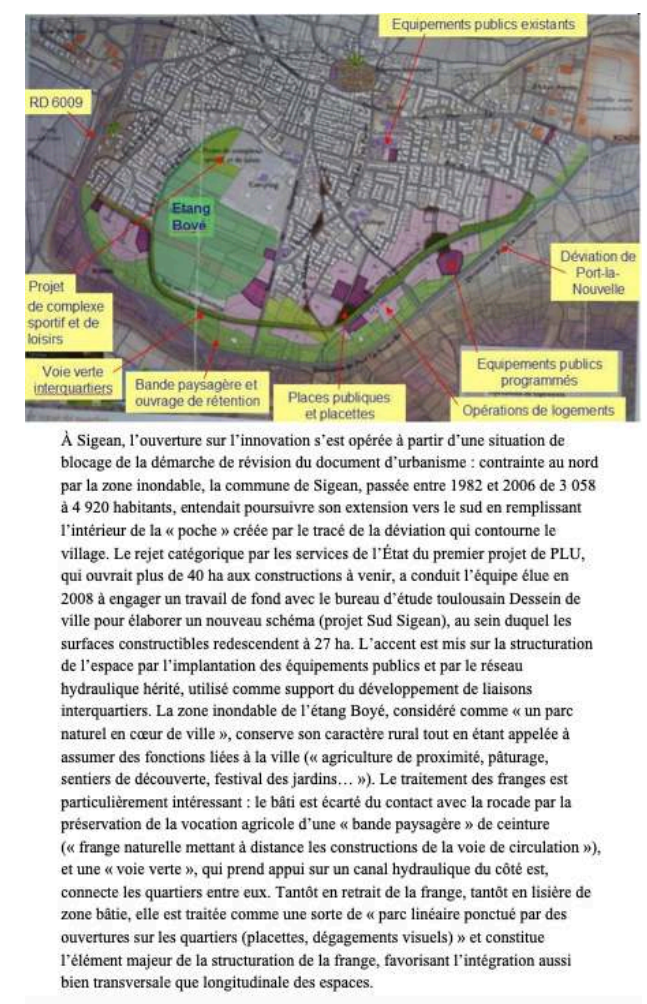

Source : ActuSigean, numéro spécial urbanisme, mairie de Sigean, 2009.

\section{Conclusion}

Si le terme de lisière urbaine apparaît et accompagne les réflexions depuis plusieurs décennies à l'échelle des grands territoires urbains et métropolitains, force est de constater que sa traduction opérationnelle peine encore à trouver localement les ressorts et les moyens de sa mise en œuvre dans des projets concrets qui viendraient qualifier ces franges au-delà de leur seule et simple fonction de limite externe et interne entre espace bâti et espace agricole et/ou de nature.

On observe certes une certaine "percolation » de l'idée dans les PLU les plus sensibles aux injonctions intercommunales (schéma directeur, Scot, charte et plan de PNR), que l'on retrouve alors dans les PADD et à travers les orientations d'aménagement des ZAC et zones à urbaniser. Cette idée a de possibles relations avec des principes d'aménagement qui sont plus anciens, et qui se sont généralisés à l'échelon communal/ local: ceux d'une vision urbanistique classique qui cherche à soigner ses bords, ses limites, sans pour autant intégrer la recherche d'une porosité et d'un dialogue entre des paysages de nature et aux fonctions différentes que traduisent les zonages des POS et des PLU.

À un niveau plus opérationnel, persiste une réelle difficulté à traduire le principe de lisière dans l'outil PLU. Une certaine inefficience s'observe dans la réalité des pratiques d'aménagement qui en découlent. Il convient néanmoins de rappeler ici que le PLU n'est pas un outil de gestion, même si le PADD propose un projet politique pour le territoire. Cela nécessite bien souvent de mobiliser d'autres dispositifs, plus 
opérationnels, qui au-delà du dessin et de l'intention permettent à la fois de gérer ces espaces selon une visée de multifonctionnalité, et de rassembler les acteurs concernés autour d'un projet. À ceci s'ajoute la question essentielle de la maîtrise du foncier, car la création de lisières au bord de la ville bâtie suppose que la maîtrise d'ouvrage dispose de surfaces aménageables qui devront être soustraites à l'un des deux espaces qu'elle est censée mettre en relation.

Enfin, outre la rareté des mises en œuvre, sous la forme d'aménagements proprement dits, ceux-ci n'intègrent presque jamais, d'une part, les pratiques des riverains production, vente et achat de proximité, promenade-cueillette, activités sportives, contemplatives, festives ou plus triviales (sortir le chien...); et, d'autre part, leurs représentations, qui mêlent les idées de nature, de calme, d'esthétique, d'espace de liberté mais aussi souvent leur méconnaissance - parfois leur défiance ou leur hostilité - de cet autre, qu'il soit urbain, agricole ou naturel.

L'ensemble de ces questions interroge la place et le rôle des concepteurs paysagistes et urbanistes dans les projets élaborés à l'échelle locale, où leurs compétences et leurs capacités d'innovation pourraient conduire à des aménagements, porteurs d'une ambition à la hauteur de la richesse du concept de lisière.

\section{BIBLIOGRAPHIE}

Bonin, S., " The concept of urban lisière and its application in Reunion Island», dans Lambertini, A. (dir.) The role of open spaces in the transformation of urban landscapes, Bologne, Editrice Compositiri, Il Faro, 2013a, p. 211-226.

Bonin, S., « Patrimonialisation des paysages de grandes cultures : la Plaine de Versailles et son association patrimoniale ", L'Espace géographique, t. 42, nº 3, 2013b, p. 227-240

Bonnefoy, S., « La politisation de la question agricole périurbaine en France : points de repère ", Urbia. Les cahiers du développement urbain durable, n 12, juin 2011, p. 17-38.

Clément, G., Manifeste du Tiers paysage, éditions Sujet/Objet, coll. « L'autre Fable », Paris, 2004.

Courcy, C. de, Bordreuil, J.-S., « Le développement durable : une préoccupation ordinaire de l'exercice paysagiste ? », dans Luginbühl, Y., Terrasson, D (ed.), Paysage et Développement durable, Versailles, éditions Quae, 2012, p. 89-97.

Denizeau, C., « Le nouveau PLU issu de la loi Grenelle II : densifier, sans s'étaler ! », Métropolitiques , 2011, URL : http://www.metropolitiques.eu/Le-nouveau-PLU-issu-de-la-loi.html.

Dérioz, P., « La place du paysage dans l'action des parcs naturels régionaux depuis la loi Paysage (1993) : enjeu central mais mission impossible? », Projets de Paysage, $\mathrm{n}^{\circ}$ 9, décembre 2013, URL : http://www.projetsdepaysage.fr/fr/

la_place_du_paysage_dans_l_action_des_parcs_naturels_regionaux_depuis_la_loi_paysage_1993_. Desvignes, M., « Le paysage en préalable », dans Masboungi, A., Barbet-Massin O., Le Paysage en préalable, Michel Desvigne. Grand prix de l'urbanisme 2011, Marseille, Éditions Parenthèses, 2011, p. 60-61. 
Donadieu, P., Les Paysagistes, Arles, Actes Sud, 2009.

Donadieu P., Campagnes urbaines, Arles, Actes Sud, 1998, p. 224

Folléa, B., « Le paysage comme relation », Les Carnets du paysage, $\mathrm{n}^{\circ}$ 21, 2011, p. 191-213.

Goze, M., «La stratégie territoriale de la loi SRU », Revue d'économie régionale \& urbaine, $\mathrm{n}^{\circ} 2002-5$, 2002, p. 761-776.

Goze, M., « 8 - La loi d'orientation foncière : de la croissance urbaine à la métropolisation », dans Annuaire des collectivités locales, vol. 19, n 1, 1999, p. 101-121. URL: http://www.persee.fr/web/ revues/home/prescript/article/coloc_0291-4700_1999_num_19_1_1331.

Guttinger, P., " Approche du paysage en droit français ", Cahiers d'économie et sociologie rurales, $\mathrm{n}^{\circ}$ 84-85, 2007, p. 12-60, URL: http://ageconsearch.umn.edu/bitstream/189739/2/84-85-12-60.pdf

Labat, D., Aggeri, G., « La loi paysage a-t-elle eu un impact sur la planification territoriale ? », Projets de paysage, $\mathrm{n}^{\circ}$ 9, décembre 2013, URL : http://www.projetsdepaysage.fr/fr/

la_loi_paysage_a_t_elle_eu_un_impact_sur_la_planification_territoriale_.

Mahey, P., « La lisière, potentiel d'accueil des nouveaux espaces publics de débat », Les Annales de la recherche urbaine, $\mathrm{n}^{\circ} 82$, mars 1999.

Melot, R., « Contester au nom du paysage : les recours contre les documents d'urbanisme », Projets de Paysages, $\mathrm{n}^{\circ}$ 5, janvier 2011, URL : http://www.projetsdepaysage.fr/fr/

_contester_au_nom_du_paysage_les_recours_contre_les_documents_d_urbanisme\#.

Raymond, R. (coord.), Béringuier, P., Bonin, S., Darly, S., Dérioz, P., Fourault-Caüet, V., Germaine, M-A., Loireau, M., Milian, J., Noël, B., Temple-Boyer, E., Toublanc, M., « Les paysages des franges périurbaines, transitions ou parois de verre ? », dans Luginbühl, Y.(dir.), Biodiversité, paysage et cadre de vie. La démocratie en pratique, programme " Paysage et Développement durable 2 », MEDDE/Victoires éditions, Paris, 2015, p. 71-89.

Renard, V., « Grenelle II : la fin de l'étalement urbain ? », Métropolitiques, 2011, URL : http:// www.metropolitiques.eu/Grenelle-II-la-fin-de-l-etalement.html.

Thibault, C. (dir.), Legenne, C., Arbelbibe Lete, L., Comment traiter les fronts urbains ? Les Carnets pratiques du Sdrif, n 3, IAU Île-de-France, mars 2010.

Toublanc, M., Bonin, S., « Planifier les trames vertes dans les aires urbaines : une alliance à trouver entre paysagisme et écologie », Développement durable et territoires, vol. 3, n² 2, juillet 2012, URL : http://developpementdurable.revues.org/9347.

Vigny, A, Latitude Nord. Nouveaux paysages urbains, Arles/Versailles, Actes Sud/ENSP, 1998, 197 p.

\section{NOTES}

1. PFP-RIO, 2012-2015, coordination Richard Raymond - Ladyss/Larep/Gecko/Geode/Espace-Dev, financé par le Medde dans le cadre du programme « Paysage et développement durable-2 ».

2. Question en débat dès le début des années 1980, codifiée en 1995 par l'article L. 111-1-4 du code de l'urbanisme, issu de la loi Barnier (loi n 95-101 du 2 février 1995, art. 52 dit «amendement Dupont »).

3. Loi Alur, titre IV, chapitre 3.

4. Il est vrai plutôt rattachée à la problématique des infrastructures de transport par le document-cadre "Orientations nationales pour la préservation et la remise en bon état des continuités écologiques » annexé au décret portant adoption de ces mêmes « orientations ». 
5. On peut citer à titre d'exemple les publications des agences d'urbanisme et/ou de communautés urbaines de l'île de France, de Marseille, de Strasbourg, de Mulhouse, de Bordeaux...

6. Livre vert de la Plaine de Versailles, document d'étude de la préfecture de la région parisienne, 1975 , p. 8. La Plaine de Versailles était un territoire pilote pour les réflexions sur les zones naturelles d'équilibre du SDAURIF de 1976.

7. Ibid., p. 28.

8. Agence Folléa-Gautier, zone UP, Atelier de l'Isthme, 2009, Schéma intercommunal d'aménagement des lisières urbaines, territoire côte ouest, TCO, Réunion - direction de l'Aménagement.

9. Agence Folléa-Gautier, Marc Marcesse (CETE Méditerranée), 1997, Étude préalable au plan de paysage de l'Ouest, ministère de l'Équipement, DDE de la Réunion, Diren de la Réunion.

10. La $1^{\text {re }}$ tranche de 600 logements, 2500 à terme, se déployant sur 89 ha de savane.

11. 46600 personnes en plus (+59\%) depuis $1975-+2090$ personnes par an entre 1999 et $2006-$ pour le territoire de la communauté d'agglomération créée en 2002, qui comptait 125500 habitants en 2014.

12. Agence Ici et là Paysage/Aspay, document provisoire, 2012.

\section{RÉSUMÉS}

À partir d'une analyse croisée d'un corpus de documents d'urbanisme élaborés à différents échelons, de discours de concepteurs urbanistes et paysagistes développant l'idée de créer des lisières urbaines, et d'enquêtes de terrain dans trois régions françaises, cet article explore la prise en compte et les différents sens donnés à la zone de contact entre l'espace urbain bâti et l'espace naturel et agricole qui l'environne. La perspective est à la fois historique, notamment pour le cas de la région parisienne, et géographique, avec des terrains franciliens, d'outre-mer (côte ouest de la Réunion) et languedociens (Narbonnaise). Comment la notion de lisière urbaine, imaginée par des professionnels de la conception de l'espace et mise en avant récemment dans de nombreux projets, vient-elle renouveler la manière de penser l'aménagement et la gestion des franges urbaines, et percole-t-elle sur le terrain? Si l'idée de créer ces lisières s'est bien diffusée dans les documents d'urbanisme, notamment au-dessus de l'échelon local, sa traduction opérationnelle est loin d'être acquise et révèle de nombreux écarts entre discours et réalité.

Based on a cross-analysis of the body of different urban planning documents, the discourses of urban and landscape designers developing the notion of the urban fringe, and field studies in three French regions, this article explores the different meanings associated with the contact area between the built up urban space and the natural and agricultural spaces surrounding it. The perspective is historical, namely in the case of the Paris region, and geographic in the cases of sites in the Ile-de-France region, overseas (East coast of Reunion island) and in the region of Languedoc (Narbonne). How does the notion of the urban fringe conceived by urban and landscape designers, which has recently been focused on in many projects, change the way of thinking in the development and management of urban fringes and does this transpire in the field? Although the idea of creating these fringe areas is becoming increasingly widespread in urban planning documents, namely at the local level, in operational terms this is far from being the case and this shows the gap that exists between the discourse and reality. 
INDEX

Mots-clés : planification, périurbain, lisière urbaine, paysagisme, Plaine de Versailles, Narbonnaise, île de la Réunion

Keywords : urban planning, peri-urban, urban fringe, landscape design, Plaine de Versailles, Narbonne, Reunion Island

\section{AUTEURS}

\section{SOPHIE BONIN}

Géographe, Sophie Bonin est maître de conférences à l'École nationale supérieure de paysage de Versailles, Larep.

s.bonin[at]ecole-paysage[dot]fr

\section{MONIQUE TOUBLANC}

Sociologue, elle est maître de conférences à l'École nationale supérieure de paysage de Versailles, Larep.

m.toublanc[at]ecole-paysage[dot]fr

\section{PIERRE DÉRIOZ}

Géographe, Pierre Dérioz est maître de conférences HDR à l'université d'Avignon et des Pays de Vaucluse, UMR Espace-Dev 228 IRD.

pierre.derioz[at]univ-avignon[dot]fr

\section{PHILIPPE BÉRINGUIER}

Géographe, Philippe Béringuier est maître de conférences à l'université Toulouse Jean-Jaurès, UMR GEODE 5602 CNRS.

philippe.beringuier[at]univ-tlse2[dot]fr 